\title{
Correction of an anterior and posterior crossbite case with a modified McNamara appliance: A case report
}

\author{
Wendes Dias Mendes ${ }^{1} \bowtie$, (Duciane Macedo de Menezes ${ }^{2}$, (D) Fábio Romano ${ }^{3}$, \\ Mírian Aiko Nakame Matsumoto ${ }^{4}$, (1) Maria Bernadete Sasso Stuani ${ }^{5}$
}

\section{Highlights}

Crossbites are frequent malocclusions in dental routine and their management by pediatric dentistry extends his/her field of work.

${ }^{1}$ Master Student, Department of Basic and Oral Biology, School of Dentistry of Ribeirao Preto, University of São Paulo, Ribeirao Preto-SP, Brazil.

${ }^{2}$ Full Professor, School of Health and Life Sciences, Pontifical Catholic University of Rio Grande do Sul, Porto Alegre- RS, Brazil.

3 Associate Professor, Department of Pediatric Dentistry, School of Dentistry of Ribeirao Preto, University of São Paulo, Ribeirao Preto-SP, Brazil.

4 Associate Professor, Department of Pediatric Dentistry, School of Dentistry of Ribeirao Preto, University of São Paulo, Ribeirao Preto-SP, Brazil.

5 Associate Professor, Department of Pediatric Dentistry, School of Dentistry of Ribeirao Preto, University of São Paulo, Ribeirao Preto-SP, Brazil.

\section{Correspondence:}

Department of Basic and Oral Biology, School of Dentistry of Ribeirao Preto, University of Sao Paulo,

Ribeirao Preto, SP, Brazil.

E-mail address:

wendesorto@usp.br

Received: 31 January 2021

Accepted: 2 March 2021

Online First: 22 March 2021
Based on the correct diagnosis of crossbites, the clinician can manage more effective and less timeconsuming treatments, leading to patient satisfaction and increasing their self-esteem.
This work allows the pediatric dentist to be familiarized with the smart treatment of combined posterior and anterior crossbite and encourages $\mathrm{him} /$ her to treat them.

\section{Abstract}

Anterior and posterior crossbites are malocclusions in the sagittal and transversal dimensions, respectively. As self-correction is rare in these alterations, early interception is recommended to allow normal occlusion and facial development. This case report aimed to discuss the treatment of an eight-year-old boy with an increased inferior facial third, who was submitted for rapid maxillary expansion with a modified bonded appliance to solve both transversal and anteroposterior deficiencies. The correction of both malocclusions was achieved within 21 days. The advantages of this procedure were to gain space in both arches, enlarge the maxillary arch, and improve nasal breathing. The interceptive therapy, as well as a well-planned appliance, proved to be effective and important for retrieving the patient's normal condition and quality of life.

Keywords: Interceptive Orthodontics; Crossbites; Malocclusions; Treatment 


\section{INTRODUCTION}

Early orthodontic treatment can be described as the intervention in either primary or mixed dentition to allow normal occlusion and skeletal development before the establishment of the permanent dentition. ${ }^{1}$ Crossbites are malocclusions that frequently occur in children and whose prevalence are seen in the literature ranging from $8-22 \%$ relating to posterior crossbite and 2,2- $12 \%$ to anterior crossbite..$^{2-3}$

Posterior crossbite is any atypical buccallingual relation between opposing arches in centric occlusion. Its occurrence can be attributed to impaired nasal breathing, muscular dysfunction, sucking habits, genetic factors, certain swallowing habits, chronic mouth breathing, traumas, and obstructive sleep apnea syndrome (OSAS).2,4,5 On the other hand, anterior crossbite is characterized as the reversed labiolingual relationship of the incisors, where the incisal edges of maxillary anterior teeth are located backwardly to those of the mandibular ones. ${ }^{3}$ Its etiological factors are described as skeletal dysplasia, where the mandible is positioned anteriorly to the maxilla, acquired muscular reflex pattern of mandibular closure, and a decrease in the axial inclination of one or more maxillary incisors. In some cases, this occlusal alteration is associated with Class III malocclusion. ${ }^{3-6}$

Spontaneous correction of crossbites is extremely unusual, therefore, early interceptive interventions are required. ${ }^{5}$ Consequences related to delay in the correction of the posterior crossbite for permanent dentition are reported as unpaired facial growth leading to asymmetry, altered muscle function, and temporomandibular joint dysfunction. ${ }^{2-5}$ Besides, the negative outcomes related to the anterior crossbite are gingival recession, loss of alveolar bone support, and mobility of the lower incisors, along with potential adverse growth influences on the anterior portion on the maxilla. ${ }^{7-8}$
Both abnormalities can be classified into three types regarding the etiological factor: skeletal, functional, and dental. ${ }^{2}$ One of the most common treatments to correct the normal transverse maxillary deficiency is the Rapid Maxillary Expansion (RME), which can be performed with different appliances. ${ }^{9}$ The correction of anterior crossbite depends on its specific type, and there is a range of treatment options. ${ }^{3}$ When the etiology is not skeletal, fixed or removable appliances can be used in the primary or late mixed dentition. However, if there is a skeletal bone implication, a reverse pull facemask is indicated. ${ }^{10}$

This case report aimed to present and offer to pediatric dentists a simple and time-saving clinical treatment option for the anterior and posterior crossbites simultaneously.

\section{CASE REPORT}

An eight-year-old boy with no notable medical history presented to the Preventive and Interceptive Clinic of our Dentistry Faculty with the chief complaint of "crooked" maxillary anterior teeth. He presented a symmetric pattern at the facial examination, competent lip sealing, obtuse nasolabial angle, good chin-neck line, convex profile, and an increased lower anterior facial third. The functional examination revealed mixed breathing with oral predominance. Written consent was taken for this case report.

The intraoral examination showed the patient was undergoing the inter-transitional period of mixed dentition with the presence of all primary teeth, Class I molars, canine relationships, and negative overjet $(-2 \mathrm{~mm})$, positive overbite, and no deviation of the midline (Figure 1). $\mathrm{He}$ presented a narrow maxilla with an ogival palate and the presence of bilateral posterior crossbite. According to Moyers mixed dentition space analysis method ${ }^{11}$ the maxillary arch presented a negative discrepancy of $-4.9 \mathrm{~mm}$ and the mandibular arch of $-1.0 \mathrm{~mm}$. 

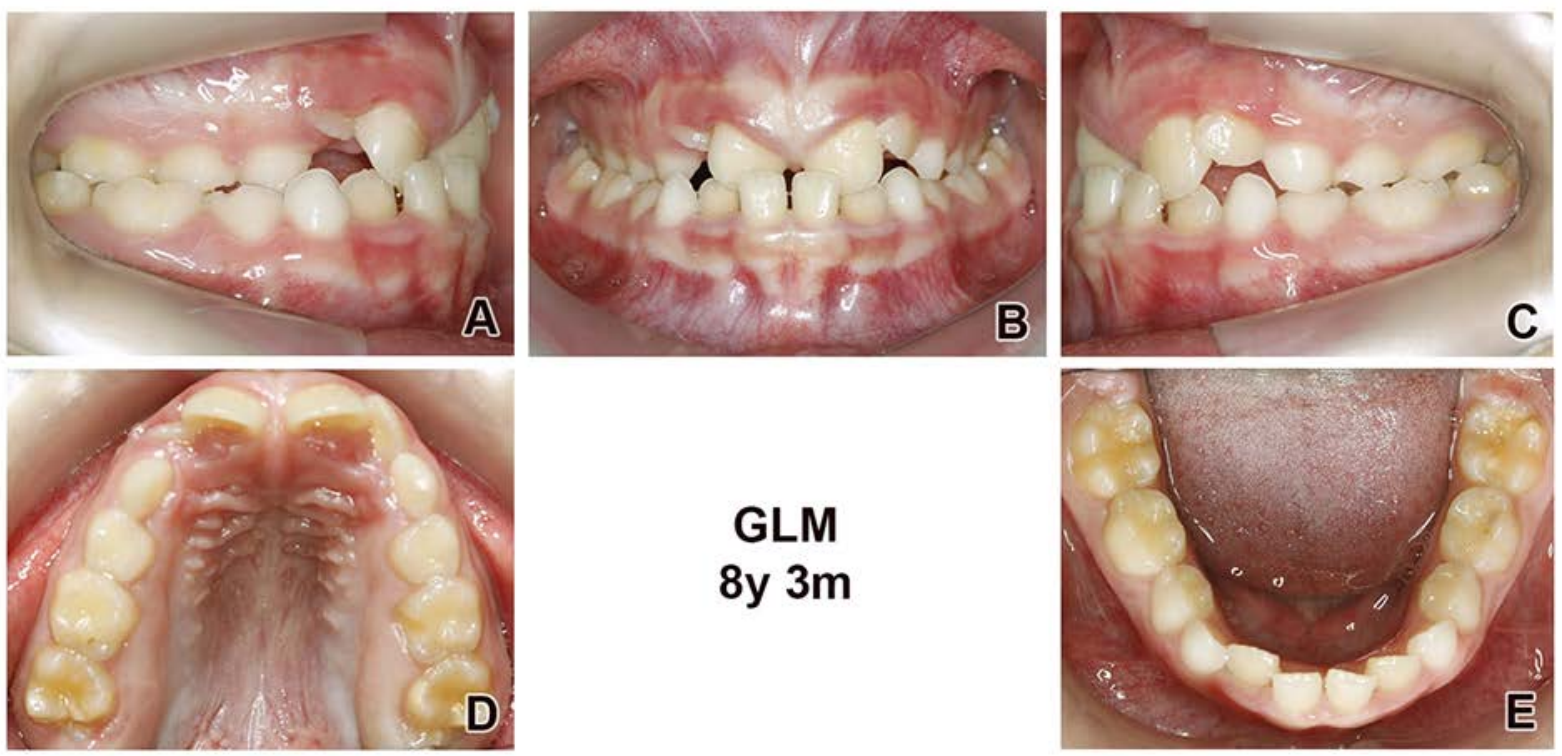

Figure 1. Pretreatment intraoral pictures. Lateral view of the right side (A). Frontal view (B). Lateral view of the left side (C). Occlusal view of the upper jaw (D) and mandibular occlusal view (E)

Panoramic radiograph (Eagle 3D, Dabi Atlante, Ribeirao Preto, SP, Brazil) showed the presence of the complete permanent teeth series, except the third molars (Figure 2.A). The Steiner's cephalometric analysis values revealed normality in the sagittal aspect $\left(\mathrm{ANB}=1^{\circ}\right)$, with an acceptable relationship of the maxilla and the mandible to each other and concerning the anterior base of the skull. However, the cephalometry (Eagle 3D, Dabi Atlante, Ribeirao Preto, SP, Brazil) also showed that the patient had a predominant vertical growth pattern of the mandible (SN.GoGn $=40^{\circ}$; SN.Gn $=70^{\circ}$ ) and that his maxillary incisors were retruded and retroclined $\left(1-\mathrm{NA}=2 \mathrm{~mm} ; 1 . \mathrm{NA}=15^{\circ}\right)$ (Figure 2.B).
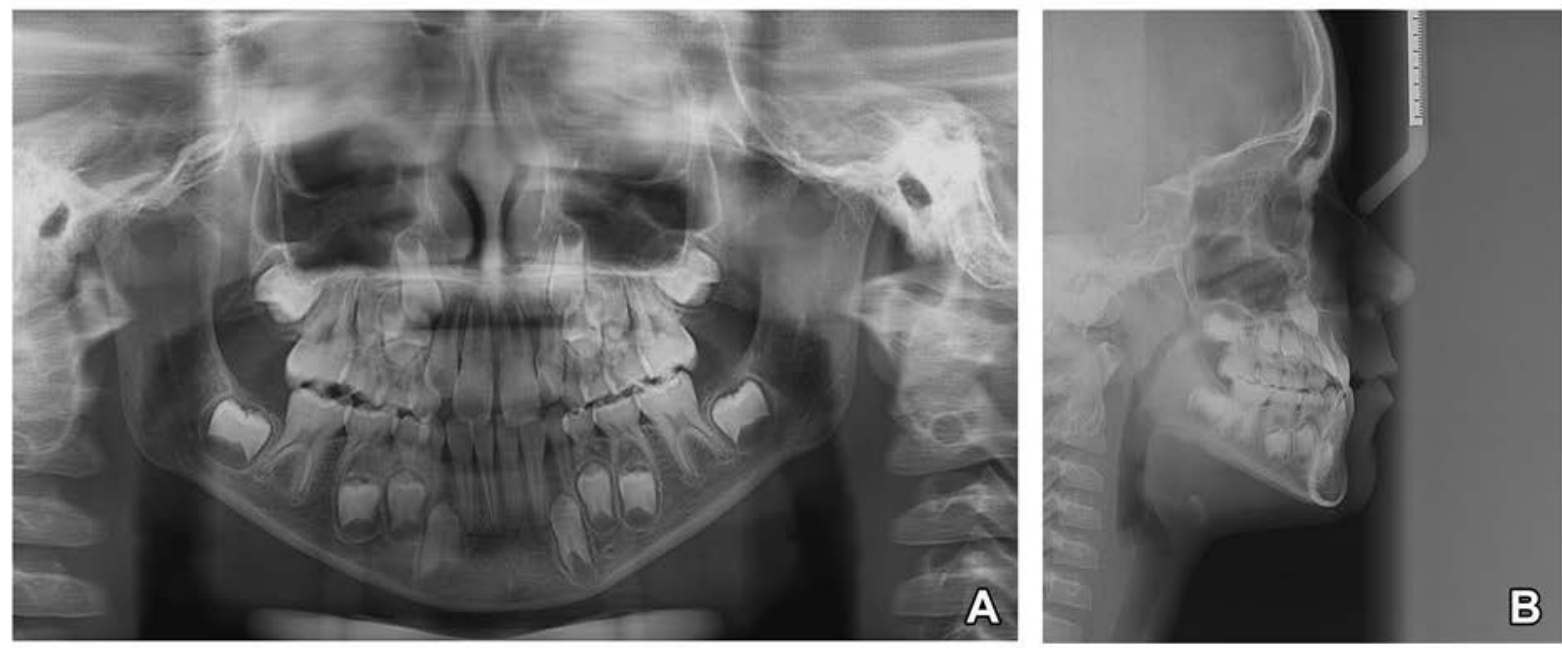

Figure 2. Pretreatment radiographic documentation. Panoramic radiograph (A). Lateral teleradiography (B) 
Based on the presented data, the patient was diagnosed with bilateral skeletal posterior crossbite and dental anterior crossbite. Interceptive treatment was planned to re-establish normal patterns.

Considering all characteristics of the malocclusions and aiming to provide a less timeconsuming treatment since both problems could be corrected at the same time, a modified expander device, with digital springs, was proposed. A dental cast was obtained through the impression of the superior dental arch with alginate (Zhermack, Badia Polesine, RO, Italy). This modified McNamara RME appliance with a palatal split screw with $11 \mathrm{~mm}$ expansion range (Reference: 65.05.012, Dental Morelli, Sorocaba, SP, Brazil) and an occlusal splint made of selfcuring acrylic resin (Classico, Campo Limpo Paulista, SP, Brazil) with two double-helix digital springs, made of stainless steel 0,020 inches (Dental Morelli, Sorocaba, SP, Brazil) for the projection of the incisors, was placed in the maxillary arch with glass ionomer cement (SSWhite Duflex, Vasco da Gama, RJ, Brazil) (Figure 3).

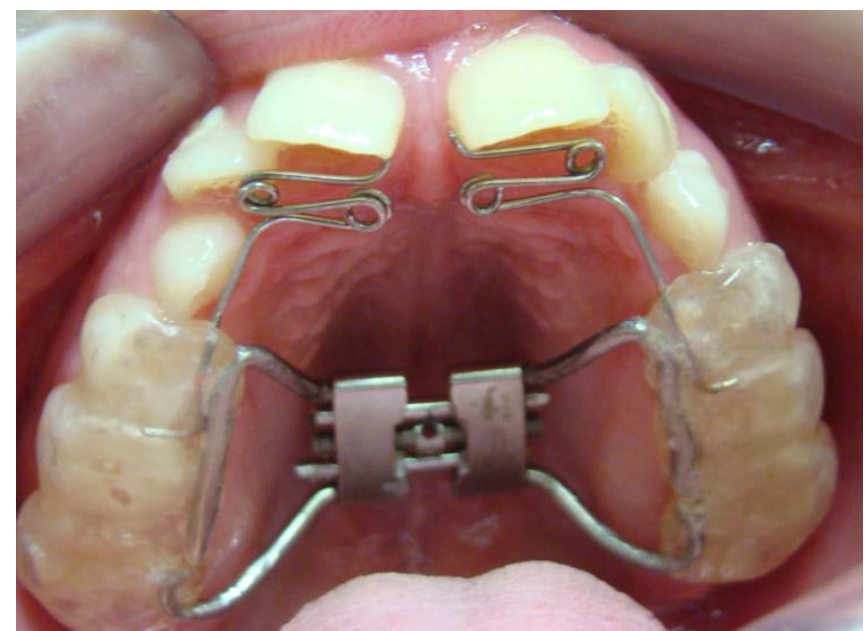

Figure 3. Modified McNamara appliance with two double-helix digital springs
The activation protocol followed the classical recommendation by Haas $(1961)^{12}$ consisting of 4 $1 / 4$ turn activations of the screw on the bonding day and the parents were oriented to perform $2 \frac{1}{4}$ turn activations for the following days, one in the morning and the other in the evening. Each activation opened the screw by $0.25 \mathrm{~mm}$, totaling $0.5 \mathrm{~mm}$ per day. Instructions on how to clean the appliance were given to the patient (at the same time with transversal expansion). He returned weekly for monitoring the orthopedic and dental effects. The double-helix digital springs were activated only once.

The appliance was activated for 21 days until overcorrection had occurred when the palatal cusps of the maxillary first molars were occluding on the buccal cusps of the mandibular first molars. There were no deleterious effects from the use of this appliance. A positive overjet was obtained, the negative discrepancies of the maxillary and mandibular arches were solved and the patient's parents referred to an improvement in their son's breathing. Then, the screw was immobilized with self-curing acrylic resin and the appliance used passively as a retainer for 6 months. ${ }^{13}$

After the retaining period, the RME appliance was removed and a maxillary removable retainer with digital springs was placed and activated biweekly for 6 months for corrections of rotations. New orthodontic records were required and the cephalometric analysis showed the correction of the maxillary incisors and their adequate axial inclination. Treatment goals for the mixed dentition were achieved (Figure 4). Comprehensive orthodontic treatment was considered for permanent dentition. 

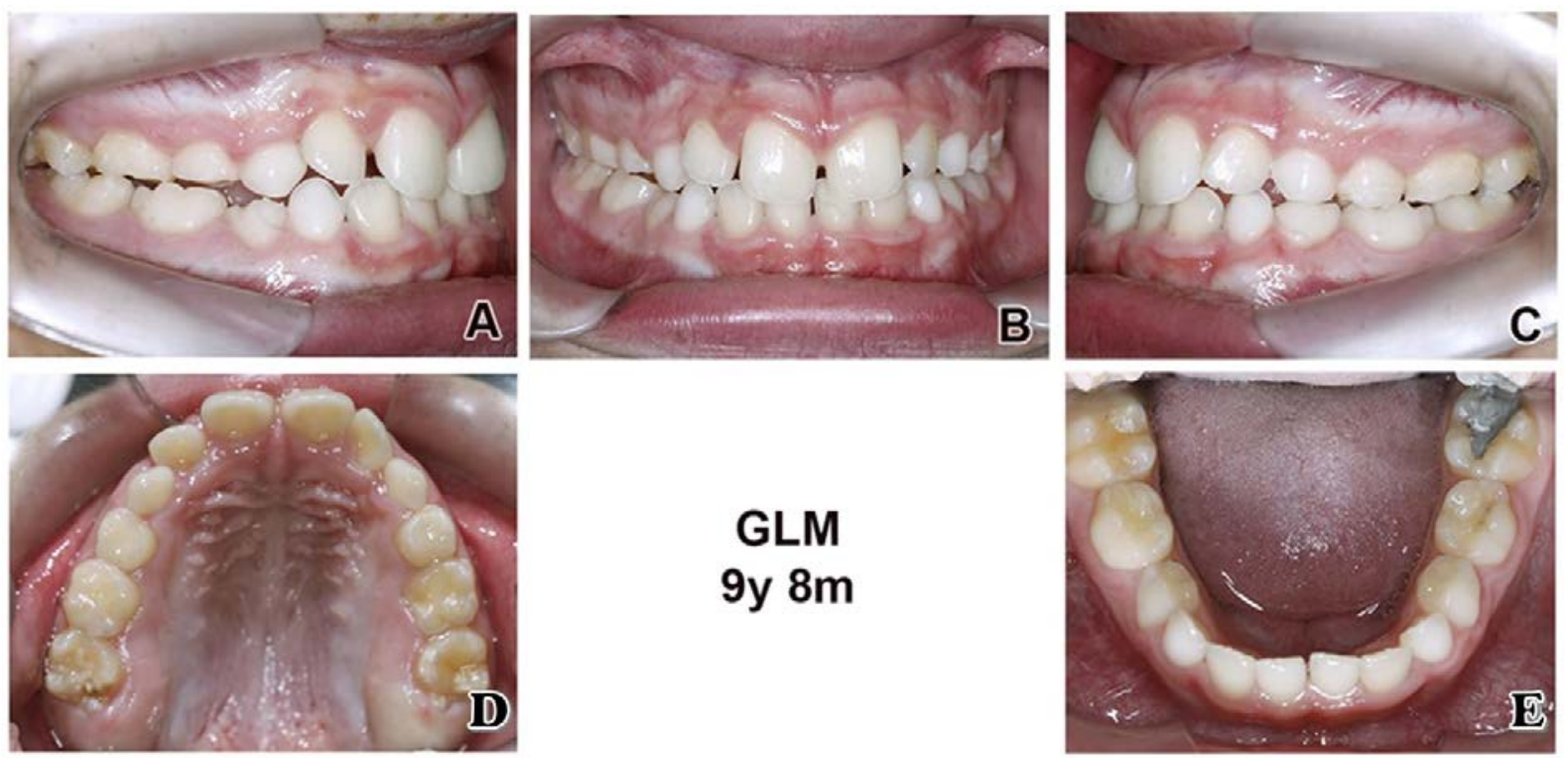

Figure 4. Intraoral aspect at the end of the first phase treatment. Lateral view of the right side (A). Frontal view (B). Lateral view of the left side (C). Occlusal view of the upper jaw (D) and mandibular occlusal view (E)

The final panoramic radiograph showed an advancement in the inferior canines' eruption path, perhaps due to the redirecting of the gubernacular canals after gaining arch perimeter. The maxillary central incisors presented an increase in root length and normal development of apicigenesis, indicating no harmful effects of the springs to these teeth. The mandibular third molars' crypts were also present (Figure 5.A).
The cephalometric analysis showed an increase in the axial inclination and protrusion of the maxillary incisors $\left(1 . \mathrm{NA}=22^{\circ} ; 1-\mathrm{NA}=4 \mathrm{~mm}\right)$ (Figure 5.B). The vertical measurements were virtually kept with the same values, with a slight elevation of only $1^{\circ}$ for both angles $\left(\mathrm{SN} \cdot \mathrm{GoGn}=41^{\circ}, \mathrm{SN} \cdot \mathrm{Gn}=71^{\circ}\right)$.
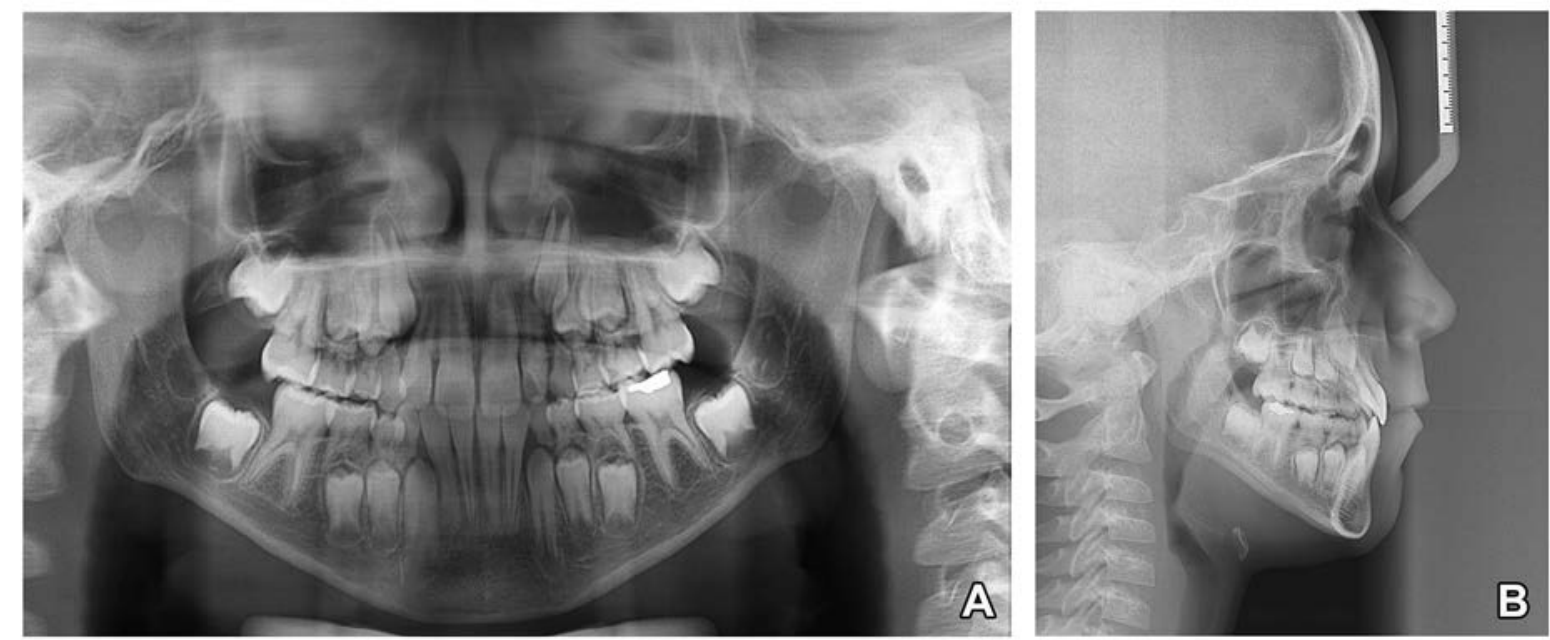

Figure 5. Post-treatment radiographic documentation. Panoramic radiograph (A). Lateral teleradiography (B) 
The final analysis of the partial maxillary superposition revealed there was no molar extrusion (Figure 6).
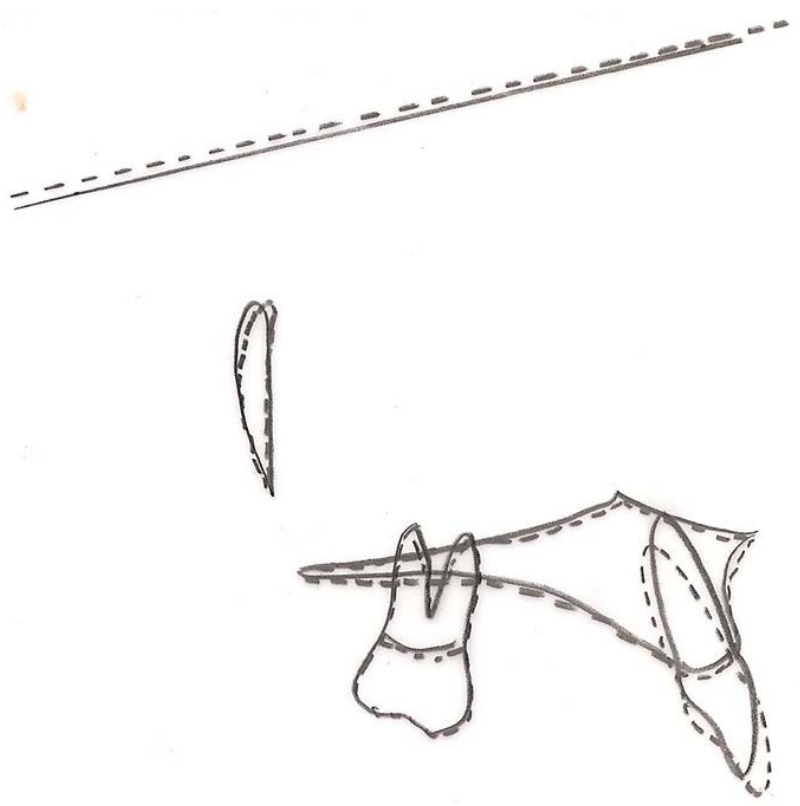

Figure 6. Partial maxillary superposition of the pretreatment (continuous line) and post-treatment (dashed line) cephalometric tracings

\section{DISCUSSION}

Pediatric dentists are commonly the first dental health care professionals to evaluate children's oral condition. ${ }^{14}$ This early evaluation is extremely important since there is an opportunity to identify incipient signs of malocclusions and intercept them. Early treatment can eliminate or decrease the gravity of developing malocclusion. ${ }^{15}$ Similarly, skeletal outcomes of considerable proportions and stability can be obtained at this phase. $^{16}$

The narrowness of the maxilla is frequently a sign of its skeletal deficiency and clinically can be identified as a posterior crossbite without deviation of the midline. Moreover, this transverse deficiency may lead to impaired nasal breathing since the maxilla is the floor of the nasal cavity. The White Paper (2019) ${ }^{17}$ proposed by the American Association of Orthodontists, suggests that RME may result in the reduction of the nasal airway resistance and an increase in the volume of the nasopharynx and nasal cavity.

When facing a posterior crossbite due to a narrow maxilla, the dentist should be able to recognize the advantages of the procedure and the possible side effects which may occur originating from the RME. Splintless RME appliances may cause extrusion of the posterior teeth, consequently promoting a clockwise rotation of the mandible. ${ }^{18}$ As our patient already presented an increased lower anterior facial third, an acrylic-splint expander appliance was used. In theory, the occlusal splint would work as a posterior bite block and prevent the vertical eruption of the posterior teeth during treatment. ${ }^{19}$ The final analysis of the partial maxillary superposition revealed there was no molar extrusion. This result showed that it was effective for vertical control.

The correction of the anterior crossbite using a modification in the appliance that was first designed by McNamara and Brudon ${ }^{20}$ was only possible due to the correct identification of the etiological factor. As the patient did not present a functional shift of the mandible and the lateral cephalometry showed a good relationship between the bone bases and a decreased axial inclination of the maxillary incisors, we were able to diagnose the anterior crossbite as a dental one. ${ }^{21}$

Bhardwaj $^{22}$ highlighted the necessity of disoccluding the bite to allow the correction of the anterior crossbite. Hence, the choice for the acrylic-splint fitted the necessity of correction for the anterior and posterior crossbites. Concerning the dental anterior crossbite, some authors recommend that the digital springs' inches of stainless steel (SS) may vary between 0.012" to 0.020 ". It may depend on how many teeth are in contact with them. Considering that the maxillary central incisors have big crowns and the force decay rate is high when using SS, we chose to use the 0.020 " length. Only one activation was 
required due to its effectiveness and, as the type of movement was as torque-like, the crowns went forward increasing the axial inclinations of those teeth. ${ }^{23-24}$

Reduction in the negative discrepancy of the maxillary arch after RME is well documented in the literature due to the increase in the perimeter of the arch.5,9,25,26,27 Furthermore, the expansion of mandibular arch widths can be explained by the presence of altered dental contacts, which could incline posterior mandibular teeth buccally. ${ }^{25}$ Our patient kept all his primary teeth during the whole treatment, not consuming the Leeway Space to solve the crowding.

When the professional keeps the benefits of early interception in mind and is concerned with mastering a good diagnosis and treatment plan for time and money-saving for his/her clinical practice, the pediatric dentist has much to gain overall.

\section{CONCLUSIONS}

Early interception is a great strategy that dentists should use to prevent many malocclusions in the future or even to avoid their worsening. The mechanics do not need expensive apparatus or complex mechanisms, nevertheless, a well-planned procedure based on the mastering of the diagnosis can present a great range of benefits not only to the patient but also to the dentist who may optimize his/her clinical work.

\section{REFERENCES}

1. Kerosuo H. The Role of Prevention and Simple Interceptive Measures in Reducing the Need for Orthodontic Treatment. Med Princ Pract 2002;11:16-21

2. Andrade AS, Gameiro GH, De Rossi M, Gavião MBD. Posterior Crossbite and Functional Changes: A Systematic Review. Angle Orthod 2008;79:380-386

3. Khalaf $\mathrm{K}$ and Mando M. Removable appliances to correct anterior crossbites in the mixed dentition: a systematic review. Acta Odontol Scand 2020;78:118-125

4. Lippold C, Stamm T, Meyer U, Végh A, Moiseenko T, Danesh G. Early treatment of posterior crossbite - A randomized clinical trial. Trials 2013;14:1-10

5. Tanaka O, Fornazari I, Parra A, Castilhos B, Franco A. Complete Maxillary Crossbite Correction with a Rapid Palatal Expansion in Mixed Dentition Followed by a Corrective Orthodontic Treatment. Case Rep Dent 2016; 2016:1-6

6. Weinberg R. The anterior crossbite: report of cases. J Am Dent Assoc 1975;90:621-624

7. Miamoto C, Marques L, Abreu L, Paiva S. Comparison of two early treatment protocols for anterior dental crossbite in the mixed dentition: A randomized trial. Angle Orthod 2018;88:144-150

8. Borrie F, Bearn D. Early correction of anterior crossbites: A systematic review. J Orthod 2011; 38:175-184

9. McNamara J, Sigler L, Franchi L, Guest S, Baccetti T. Changes in occlusal relationships in mixed dentition patients treated with rapid maxillary expansion. Angle Orthod 2010;80:230238

10. Jorge JO, Corradi-Dias L, Flores-Mir C, Pordeus IA, Paiva SM, Abreu LG. Comparison Between Removable and Fixed Devices for Nonskeletal Anterior Crossbite Correction in Children and Adolescents: A Systematic Review. J Evid Based Dent Pract 2020;20:1-10

11. Galvão M, Dominguez GC, Tormin ST, Akamine A, Tortamano A, de Fantini SM. Applicability of Moyers analysis in mixed dentition: A systematic review. Dental Press J Orthod 2013;18:100-105

12. Haas AJ. Rapid expansion of the maxillary dental arch and nasal cavity by opening the midpalatal suture. Angle Orthod 1961; 31:73-90

13. Lagravère MO, Carey J, Heo G, Toogood RW, Major PW. Transverse, vertical, and anteroposterior changes from bone-anchored maxillary expansion vs traditional rapid maxillary expansion: a randomized clinical trial. Am J Orthod Dentofacial Orthop 2010;137:304.e1312e1

14. Aldrees A, Tashkandi N, AlWanis A, AlSanouni M, Al-Hamlan N. Orthodontic treatment and referral patterns: A survey of 
pediatric dentists, general practitioners, and orthodontists. Saudi Dent J 2015; 27:30-39

15. Burhan A, Nawaya F. Preventive and interceptive orthodontic needs among Syrian children. J Egyptian Public Health Assoc 2016; 91:90-94

16. Geran R, McNamara J, Baccetti T, Franchi L, Shapiro L. A prospective long-term study on the effects of rapid maxillary expansion in the early mixed dentition. Am J Orthod Dentofacial Orthop 2006;129:631-640

17. Behrents R, Shelgikar A, Conley R, Flores-Mir C, Hans M, Levine M, McNamara J, Palomo J, Pliska B, Stockstill J, Wise J, Murphy S, Nagel N, Hittner J. Obstructive sleep apnea and orthodontics: An American Association of Orthodontists White Paper. Am J Orthod Dentofacial Orthop 2019; 156:13-28

18. De Rossi M, De Rossi A, Abrão J. Skeletal Alterations Associated with the Use of Bonded Rapid Maxillary Expansion Appliance. Braz Dent J 2011;22: 334-339

19. Wendling L, Mcnamara J, Franchi L, Baccetti T. A Prospective Study of the Short-term Treatment Effects of the Acrylic-splint Rapid Maxillary Expander Combined with the Lower Schwarz Appliance. Angle Orthod 2005;75:7-14

20. McNamara Jr, Brudon WL. Orthodontic and orthopedic treatment in the mixed dentition. 5th ed. Ann Arbor: Needham Press; 1995

21. Wiedel A, Norlund A, Petrén S, Bondemark L. A cost minimization analysis of early correction of anterior crossbite - A randomized controlled trial. Eur J Orthod 2016;38:140-145

22. Bhardwaj P, Verma SK, Rastogi K, Bhushan R. An efficient method for correction of anterior crossbite without using bite plates. BMJ Case Rep 2013:1-3

23. White TC, Gardiner JH, Leighton BC. Orthodontics for Dental Students. 3rd ed. New Delhi: Macmillan India Ltd; 1976

24. Agarwal DK, Razdan A, Agarwal A, Bhattacharya P, Gupta A, Kapoor DN. A Comparative Study of Orthodontic Coil Springs. J Ind Orthod Soc. 2011; 45:160-168

25. Melgaço C, Neto J, Jurach E, da Nojima M, Sant'Anna E, Nojima L. Rapid maxillary expansion effects: An alternative assessment method by means of cone-beam tomography. Dental Press J Orthod 2014;19:88-96
26. Lagravere M, Major P, Flores-Mir C, Orth C. Long-Term Dental Arch Changes After Rapid Maxillary Expansion Treatment: A Systematic Review. Angle Orthod 2005;75:155-161

27. O'Grady P, McNamara J, Baccetti T, Franchi L. A long-term evaluation of the mandibular Schwarz appliance and the acrylic splint expander in early mixed dentition patients. Am J Orthod Dentofacial Orthop 2006;130:202-213

\section{How to cite this article:}

Wendes Dias Mendes, Luciane Macedo de Menezes, Fábio Romano, Mírian Aiko Nakame Matsumoto, Maria Bernadete Sasso Stuani. Correction of an anterior and posterior crossbite case with a modified McNamara appliance: A case report. Contemp Pediatr Dent 2021:2(1):64-71

\section{Declarations}

Acknowledgements: Not applicable.

Conflict of Interest Statement: The authors disclose no potential conflicts of interest.

Ethics Statement: Procedure was explained to the parents and written consent was obtained for the treatment and publication of the case report.

Informed Consent: Written consent was taken for participation of parents and child in the study.

Author contributions: Conception and design: All Authors; Acquisition of data: MBSS; Interpretation of data: WDM; Drafting article: WDM; Revision artice: LMM, FLR, MANM; Final approval: All Authors

Funding: This work is not finantiated.

Data Availability: The data used to support the findings of this study can be made available upon request to the corresponding author.

Peer-review: Externally double-blinded peer-reviewed. 